\title{
Issues and Problems in Conducting Sensitive Research: A Case of HIV/AIDS in Nepal
}

\author{
Ak Narayan Poudel ${ }^{1}$, David Newlands ${ }^{2}$, Padam Simkhada ${ }^{3}$ \\ ${ }^{1}$ Public Health Researcher, Liverpool John Moores University, UK ${ }^{2}$ Staff Associate, Queen Margret University, UK ${ }^{3}$ Professor in International Public Health, Liver- \\ pool John Moores University, UK
}

Received:

11 March 2016

Revised:

12 June 2016

Accepted:

05 July 2016

\section{${ }^{\star}$ Correspondence:}

a.n.poudel@ljmu.ac.uk Public Health Researcher, Liverpool John Moores University, United Kingdom.

\begin{abstract}
The main aim of this paper is to explore the issues and problems along with possible solutions to conduct sensitive research, specifically research related to HIV/AIDS in Nepal. This paper is based on observation, information and experience obtained during research entitled, "The Economic Burden of HIV/AIDS upon Households in Nepal' and literature reviews. There are many issues and problems in conducting sensitive research. Major issues and problems are- adherence to research ethics, use of research design and sampling, and recruitment of respondents in research. The paper concluded that research on sensitive topics like HIV/AIDS is very challenging and researchers need to strictly follow ethical procedures. Maintenance of anonymity and confidentiality are the key factors for encouraging participants to become involved in such sensitive research. Similarly, a mix of qualitative and quantitative methods help to understand the complex situations encountered during sensitive research. A non-probability sampling method is preferred over other methods of sampling in such research because there is often a problem of establishing a sampling frame in populations. Similarly, support from staff from government hospitals and NGOs is crucial if people living with HIV/AIDS are to be involved in the research. The issue of incentives is a highly discussed topic in sensitive research. But, it has been concluded that incentives especially in the monetary form should not be provided in order to avoid response bias and ethical conflicts.
\end{abstract}

Keywords: HIV/AIDS, Nepal, sensitive research

Suggested citation: Poudel AN, Newlands D, Simkhada P. Issues and Problems in Conducting Sensitive Research: A Case of HIV/AIDS in Nepal. Health Prospect. 2016;15(2):5-10.

Tweetable abstract: Conducting research on sensitive topics is very challenging; however, strict adherence of research ethics is the key for the successful completion of such research.

\section{Introduction}

The researchers who conduct studies on sensitive topics need to pay more attention at every stage of their studies compared to the researchers conducting studies on non-sensitive topics. According to Lee, sensitive research is 'a research which potentially poses a substantial threat to those who are or have been involved in it' (1). This definition indicates that if there is a presence of harm to people involved in the research, including researcher and respondents, it is sensitive research. Similarly, Sieber and Stanley defined sensitive research as: 'studies in which there are potential consequences or implications, either directly for the participants in the research or for the class of individuals represented by the research' (2). In general, all research studies have some kinds of consequences. However, sensitive research often has potential effects on the personal life of respondents and sometimes on the personal security of the researcher as well (3). Possible unexpected events in sensitive topics may pose a threat, and that may raise difficult methodological and technical problems (4). Lee has divided possible threats of sensitive research areas into three (1). The first type is 'intrusive threats' which deal with the areas that are 'private, stressful or sacred'. The second type of threat is 'threat of sanction', which relates to studies of deviance and involves research which may reveal results that cause stigma and discrimination in some way. The third type of threat in sensitive research is a 'political threat', which relates to the research that may cause some sort of social conflict. According to Lee's definition, HIV/AIDS research falls into the first two categories of sensitive areas, 'intrusive threat' and 'threat of sanction' because research on HIV/AIDS can reveal private, stressful or sacred information, while if the status of the respondent becomes known to others it may cause stigma and discrimination (1).

People living with HIV/AIDS (PLHIV) often feel double stigma, first from their HIV/AIDS status and, second from their behaviour that exposed them to HIV. In Nepal, there is an understanding that HIV is transmitted if people undertake socially unacceptable behaviour like: having unsafe sex with sex workers, sharing injectable drugs and having unprotected sexual relations with people of the same sex. Stigma increases when PLHIV are deemed personally responsible for bringing it upon themselves. Therefore, PLHIV are viewed negatively by people in Nepal. Individuals with HIV/AIDS may be isolated from society and even from family members. This situation is psychologically and emotionally traumatic for the HIV infected person. They may lose their jobs or be forced to discontinue 
education and training opportunities. This situation may lead to frustration, stress, fear, guilt and might also result in extreme conditions such as suicide. Similarly, HIV/AIDS may create disharmony in community relationships. People may lose faith and trust in each other. Social values and the moral of a community as a whole may decline and result in the disintegration of society (5). Likewise, HIV is an infectious and chronic disease and therefore, people fear transmitting it from PLHIV. Due to the associated stress, threats, stigma and discrimination, PLHIV prefer to hide their status and fear taking part in research studies. Moreover, illiteracy, conservative culture, lack of awareness about the disease transmission, and association of stigma and discrimination with the disease are the important factors which make HIV/AIDS as a more sensitive issue in the Nepalese context.

Sensitivity may affect almost every stage of the research process from the formulation of a research problem to the design and the implementation of the research, and dissemination and application of the findings $(3,6)$. Therefore, it is important to investigate methodological issues and problems from the perspective of both researchers and participants. Sensitive topics also raise wider issues related to ethics, politics and legal aspects of research (1). Some examples of sensitive topics are sexual behaviour, HIV/AIDS, drug abuse, deviance, and death.

Sieber and Stanley argue that sensitive research addresses society's burning issues and policy questions (2). Therefore, raising issues and problems while conducting research on sensitive topics like HIV/AIDS is crucial. This article has been prepared in order to generate awareness, enhance knowledge and understanding, and overcome methodological issues and problems associated with sensitive research, specifically on HIV/ AIDS in Nepal.

\section{Methods}

This paper is based on observations, information and experiences gained during pilot study and main survey of the research entitled, "The Economic Burden of HIV/AIDS upon Households in Nepal'. Relevant literature was reviewed from internet search engines and text books. The literature in the Internet was searched using MEDLINE search engines (Embase- from 1996 to 2016 week 2) using different search words such as: 'sensitive research' (3 results), 'mixed methods' (372 results), 'HIV and Stigma' (30 results), and 'incentives to respondent or participant' (12 result). Altogether 35 papers ( 18 from journals and 17 from text books) were selected for the review based on the relevance to the subject matter. The selection criteria used to select the papers were: discussion on sensitive research and research ethics, discussion about incentives and its impact on research, discussion on methodological issues of sensitive research, and discussion on stigma and its impacts on research participants. An overview of the pilot study and a main survey of the research are briefly described as follows:

\section{Pilot and Main Studies}

A small pilot study was conducted in August/September 2010 to assess the validity of the questionnaires for the main survey. In the pilot study questions related to: personal information, household information, economic status, the direct cost of HIV/AIDS, productivity costs, coping strategies, and stigma and discrimination. Due to funding and time limitations, the pilot study was limited to the Kathmandu valley (Youth Vision and Nawa Kiran Plus: NGOs) and Chitwan district (Chitwan Sakriya Samuha: NGO). A purposive sampling method was employed for the study. PLHIV who were 18 years of age and above, and more than a month of HIV diagnosis were selected for the survey. PLHIV who were 18 years old and above, and more than a year of HIV diagnosis were involved in the in-depth interviews. The survey with 36 PLHIV and in-depth interviews with five PLHIV were conducted in the Pilot study.

The main study was carried out from March to July 2011. This study was conducted in six study sites in Nepal namely: BP Koirala Hospital, Sunsari; Sukraraj Tropical Hospital, Kathmandu; Bharatpur Hospital, Chitwan; Rapti Sub-Regional Hospital, Dang; Bheri Zonal Hospital, Banke; and Doti District Hospital, Doti. One treatment centre from each district was selected based on the coverage by the treatment centre, accessibility of PLHIV to the treatment centre, types of PLHIV coming to the treatment centre and the number of PLHIV coming to the treatment centres.

A survey with 415 PLHIV and in-depth interviews with 30 PLHIV were conducted in the main study, which involved both antiretroviral therapy (ART) recipients and non-recipients. Average length of the interview was around 45 minutes and it was conducted in HIV/AIDS treatment centre of the each site in the main study and NGOs' offices in the pilot study. The main difference in the settings of the pilot study and the main study was that the pilot study was solely conducted in the NGOs shelter but the main study was conducted in treatment centres of government hospitals. Treatment centres of government hospitals are the main source of HIV diagnosis, counselling and treatment to people with HIV/AIDS. NGOs are major sources of empowerment for the PLHIV and the NGOs also play a bridging role between PLHIV and the treatment centres of government hospitals (NGOs motivate PLHIV to have their treatment and encourage PLHIV to go to treatment centres from their home or shelter). The observation was conducted during the survey and in-depth interviews during pilot and main studies of the research in the above-mentioned study sites. The observation of the participants, person in charges, the volunteers of the NGOs and health professionals of the HIV/AIDS treatment centres were conducted in the study.

Issues and Problems in Conducting Research in Sensitive Topics: The Experience of Conducting Research on the Economic Burden of HIV/AIDS upon Households in Nepal

\section{Issue of Research Ethics}

Strict adherence to research ethics is crucial in sensitive research. Conducting research by luring or threatening others is not ethical. Every participant should be free to participate or not in a research. Even if an incentive is provided to the participants, they will not be compelled to answer the entire questionnaire if they do not feel comfortable. Every respondent has the right to leave the survey at any point during the questionnaire if he/she does not wish to continue. Similarly, anonymity and confidentiality must be maintained in any human research. Every piece of research needs to follow ethical principles. Adherence to research ethics is one of the major issues in conducting sensitive research.

It has been observed in the main survey and the pilot study 
that persons in charge and volunteers of NGOs are very careful about the involvement of PLHIV to any types of research studies. The persons in charge and volunteers of NGOs do not allow any researcher to involve PLHIV in their research studies by luring or threatening. The persons in charge and volunteers also come forward and ask questions about the aims and benefits of the research studies.

\section{Issue of Research Design and Sampling Method}

There is always debate on the use of research design for sensitive research. Some researchers prefer qualitative research design and some prefer mixed-methods design. It has been argued that a single research design cannot provide sufficient information about an issue in question in sensitive research $(7,8)$. Similarly, there is a debate over the use of sampling techniques in sensitive issues. The researchers who are not involved in sensitive research generally ask questions about the non-use of probability sampling technique in sensitive research (1). In fact, it is very difficult (sometimes impossible) to get a sampling frame for sensitive research. Moreover, PLHIV do not want to reveal their HIV status to other people due to fear of stigma and discrimination. Therefore, probability sampling techniques are difficult to utilise in the HIV/AIDS or other sensitive issues although it is the preferred method of sampling. If probability sampling is not possible to utilize in sensitive research, researchers need to use the non-probability sampling techniques. The non-probability sampling method does not imply the random selection of a research unit, unlike the probability sampling. Examples of nonprobability sampling include purposive sampling, convenient sampling, quota sampling, snow ball sampling or respondent driven sampling. This technique of selecting research units is highly applicable in sensitive issues such as HIV/AIDS because it is either impossible or very difficult to obtaina correct sampling frame of the population.

The author experienced that the reviewer from Nepal Health Research Council was asking a question about the non-use of the probability sampling method in this research study. Similarly, other intellectuals who were encountered in the study sites and government offices often asked the same question. They considered that we could use the name lists of PLHIV from treatment centres for the random sampling. This is not appropriate because PLHIV generally hide their identity.

\section{Problem of Recruiting Respondents}

Recruiting respondents was the biggest problem encountered by the researcher while conducting research on HIV/AIDS. There were a number of factors associated with the problems, which are described hereunder.

\subsection{Fear of Stigma and Discrimination}

Stigma and discrimination are two different terms. Stigma is related to beliefs and attitudes, while discrimination is related to actions. Observations during field studies, and results of interviews and surveys, showed that all categories of PLHIV have fear of stigma. However, the magnitude of fear of stigma is different from PLHIV to PLHIV. The PLHIV who are less exposed to the training programmes organised by NGOs/INGOs and government generally fear more with stigma. It is observed that the respondents from rural areas are also more scared to reveal their HIV status to the other people due to the fear of stigma. Similarly, it was found that respondents tend to lie to their neighbours, saying that they went shopping instead of revealing the fact that they went to a hospital for a routine check-up.

Most of the PLHIV do not reveal their HIV status to others except close family members or close friends due to the fear of possible stigma and discrimination. They think, if they take part in the research, their status will be known to other people. Therefore, the fear of stigma is the main factor that hinders the PLHIV from taking part in the research or other types of programmes. Such stigma and discrimination have mainly been fuelled in society by illiteracy, lack of knowledge about HIV and its mode of transfer, and conservative culture (9-11).

\subsection{Issue of Incentives}

Experience from the pilot study showed that it is very easy to recruit PLHIV by giving them incentives. In the pilot study, all respondents were paid for their time and efforts. The incentive amount was fixed after negotiating with the person in charge of NGO. The benefits of the monetary incentives provided to the respondents were: respondents could be recruited easily, there was no need to build a strong rapport with them and it took less time to conduct the survey as little effort was required to convince them. However, incentive may cause bias in the research due to biased responses and it also may pose ethical conflicts if its appeal is too strong (i.e. coercive) $(12,13)$. Respondents generally give the answers that the researcher wants to hear if incentives are provided to them. There would be a tendency to attract economically weak PLHIV to the research compared to economically strong PLHIV(14).

From the pilot study and the main survey, it has been found that implementation of surveys in NGO shelters was impossible without the permission of the person in charge of NGO. Generally, the person in charge of NGO negotiates the incentives in return for the time and effort given by the PLHIV to any research activities. They ask the researcher what their PLHIV would get if they took part in the research. If they agree with the incentives provided to the PLHIV, they allow the researcher to conduct the survey. But, without incentives, it is very difficult to approach PLHIV in NGOs.

\subsection{Issues of Anonymity and Confidentiality}

It was found that PLHIV always wanted confidentiality. They are scared about possible breaches of confidentiality by the researchers. They needed to be reassured by the researcher and staff working in the treatment centre or staff working in the NGOs that confidentiality would be maintained during the research. Similarly, while obtaining informed consent, the verbal or informal consent form was found to be more comfortable than the written consent form by the PLHIV as they wanted anonymity and did not want to reveal their names even to the researchers.

PLHIV were found to be concerned about the place or environment of the interview during the research. They felt comfortable in a closed room rather than in an open area for the interview. This was mainly due to the fear of breaching confidentiality to other people regarding their HIV status. In developing countries like Nepal, there is a problem with even getting a proper room in which to conduct an interview. Sometimes, this problem reduces the level of participant involvement and increases the refusal rate. This was experienced more in HIV/ AIDS treatment centres (government hospitals) than in the NGO's shelter. 


\subsection{Extensively Researched Area}

It was found that respondents were frequently approached by many NGOs, INGOs and other researchers. NGO's in-charges, volunteers for the treatment centres, and the respondents themselves reported that many people came to them wanting to conduct research about them on different issues. They claimed that none of the researchers worked in their favour or helped them to improve their livelihoods. Therefore, they were tired of giving their information to the researchers. For this reason, PLHIV do not want to take part in research studies. It was found that, being an important public health issue, many researchers, NGOs, and INGOs want to conduct research and launch other programmes for PLHIV. This made them tired of repeating the same story to many people.

\section{Discussion}

One of the most important issues in sensitive research is research ethics. 'Research ethics' refer to rules of morally good conduct for researchers. Research ethics are based on moral and political beliefs, which are external to the research itself (15). Research ethics are defined as the standard practices for privacy and confidentiality protection for human subject participants (12). Before conducting a survey or interview, participants must be informed that their participation is voluntary, they will not be penalized if they refuse or leave the survey and they may skip any question if they do not feel comfortable answering or discontinue their participation at any time. Incentives provided by the researcher do not reverse their right to skip certain questions or end their involvement in a study if they wish. Moreover, Valerio and Mainieri argue that incentives should never be used to pressurize a respondent into participating in a study or to answer a specific question if they really do not want to do so (12). The researchers should not force, threaten, pressurize, or trick the potential respondents while conducting the survey. In research, human subjects have the right not to be abused physically or psychologically. They have the right of privacy and protection of their status. Now, every research conducted on human subjects must be approved by the human subjects review board' (16).

In human research, consent forms are generally used to obtain permission from a parent or guardian of a child or adolescents (below 18 years of age) who has the legal right to give permission for someone in her or his charge to participate in research. However, in some research an adult ( $\geq 18$ years of age) may be asked to sign a consent form about his or her own agreement to co-operate. Some research of sensitive topics relies on confidentiality to increase the likelihood of those respondents answering questions honestly. However, confidentiality might be compromised if a respondent signs a consent form and names are subsequently linked to answers. Due to this possibility, Institutional Review Boards (IRBS) (or Ethical Review Committee located in the USA) generally waive the need for a consent form and a signature for surveys and allow the informed consent process to occur informally as part of the survey itself (17).

Stigma and discrimination is commonly associated with HIV/AIDS because these are related to issues such as sex, drug use and death. Both terms are based on negative views of people towards others, simply because they are seen as belonging to a particular group. Some of the previous studies have demonstrated that a lack of understanding about HIV/AIDS and misper- ceptions about HIV transmission routes are important factors contributing to stigma and discrimination (18-20). Similarly, other research studies have shown that experiences of stigma have negative impact on the psychological well-being of people living with HIV/AIDS. Certain manifestations of HIV stigma in particular settings impact the psychological well-being of people living with HIV/AIDS more than other (21). HIV-related stigma can hinder HIV prevention efforts, inhibit treatment adherence, function as a barrier to HIV testing and negatively impact social relationships and psychological well-being of people living with HIV/AIDS $(18,22,23)$.

HIV-related stigma is a social phenomenon due to which an HIV infected individual is considered as tainted, spoiled or flawed by others (22). It weakens social interaction, community support and strength by creating social distance among PLHIV from other parts of society (24). The manifestation of HIV/AIDS stigma not only varies with cultural and national setting, but also by whether one is considering intrapersonal versus societal levels of stigma (23). Nepalese people perceive HIV/AIDS as a bad person's disease and they think it is due to bad deeds from the past. Therefore, HIV illness is perceived as a punishment for some wrong-doing. People think personal habit and behaviour are more responsible for HIV than the situational one. Similarly, Nepalese culture is not open to discussion about sex and sexuality and the people are dissuaded from speaking about sexual matters with elder people or other responsible family members. HIV infection is considered to be a result of deviant behaviour (25). Due to such a culture in Nepal, PLHIV feel stigma, fear and helplessness and do not disclose their HIV status to other people. Such stigma not only hinders control and prevention of HIV infection, but also discourage HIV infected people from taking part in HIV related research and programmes.

In our study, fear of stigma and discrimination was found to be one of the major problems of involving respondents in HIV/ AIDS research. They were always worried about possible stigma and discrimination and did not want to expose themselves as HIV positive towards other people.

In most research, incentives are not provided to the participants. However, some researchers provide incentives to thank participants for their contributions or for motivating respondents for participation (12). Some authors argue that monetary payments are often used as inducements and they motivate people to do something $(26,27)$. Monetary payment has positive effects on respondents' willingness to participate in any kind of research regardless of the risk. However, payment has some influence on respondents' potential behaviour regarding concealing information about restricted activities (drug use, prostitution, gambling etc.) (28). Gates et al. argue that monetary incentives could be an effective way to increase the number of postal questionnaires returned and reduce loss to follow-up in clinical trials (29). From normative ethical perspectives, various authors have argued for and against the use of monetary payments to research participants (30). Grady argues that inducements are offers not threats (27). But, Macklin argues that monetary inducement encourage people to expose themselves to risk and such inducements increase inequality in the research process because the majority of research participants would be from lower socioeconomic status (14). Many researchers worry that incentives might cause response bias.

The possibility of response bias has been evaluated in a review paper by Massey et al., where the paper compares respons- 
es from those respondents who receive an incentive to those respondents who do not (31). It has been found that differences between incentive groups and non-incentive groups are not statistically significant. But, a few other studies have found that incentives may produce slightly more favourable evaluations or positive ratings from incentive groups compared to nonincentive groups (12). Palmers argues that inducement can be undue only in research studies that involve the possibility of the highest form of physical or psychological injuries and risks (32). Incentives can help to increase response rates; however, they can add significant cost to the study (33). There would be a significant possibility that economically weak respondents would be attracted to the study. Therefore, we suggest not to provide monetary incentives to the respondents.

Confidentiality in a research refers to the methods for protecting the collected information. The confidentiality of the information is expected by both researchers and respondents. It is important for the success of a piece of research because research participants would be much more willing to participate if they thought the researcher would maintain confidentiality in the research (34). Anonymity protects the privacy of the subjects studied. It is also assumed that where the human subjects in the research cannot be identified they cannot be harmed by data about them appearing in the local area. However, the identification of research participants can sometimes be predicted from published material if sufficient care is not taken to protect confidentiality (1). Researchers must promise that research sites and subjects' identities will remain confidential (35). Anonymity is important for the success of research because it can help to protect the privacy of the information provided by the respondents. Anonymity can therefore improve co-operation when the research poses exceptional risks for participants (36).

Surveys of sensitive issues like HIV/AIDS, drug abuse, gambling and others may benefit from anonymity of the respondents. When a survey seeks to get information about sensitive questions, anonymity is likely to improve the chance of obtaining information on stigmatizing behaviour or unpopular attitudes and views. However, anonymity hinders follow-up research and also causes difficulty in verifying the information with respondents after the research (34). Anonymity and confidentiality should be maintained in every type of sensitive research to improve respondents' participation.

As a major public health issue, HIV/AIDS is a main focus for research and programme activities in Nepal. In the threeyear National Plan (2008-2010) of Nepal, HIV/AIDS was accorded "priority 1" health issue status. Therefore, government, NGOs, INGOs and private sectors are targeting the PLHIV for their programmes, research and studies. There are around 400 NGOs and 60 INGOs working for the PLHIV in Nepal (37). Similarly, large numbers of students conduct research on HIV/ AIDS every year. Therefore, PLHIV were approached extensively by government, NGOs, INGOs, researchers and students for different purposes. PLHIV could see the direct benefit of the programmes launched by NGOs/INGOs and government but not the research projects. Therefore, they prefer immediate direct benefit rather than indirect long-term benefit in the future.

\section{Conclusion}

Research over the past three decades has developed better methodological understanding of conducting research on sensitive issues. But, there are still a number of unresolved theoretical and methodological problems. Conduct of research on sensitive topics like HIV/AIDS is very challenging. Sensitive research should be conducted in a most thoughtful and careful manner. To develop a broad understanding of the issues in sensitive research, it is important to examine more than just the consequences of undertaking the research. The following conclusions are drawn based on the findings and discussions of the paper:

- The researchers working on sensitive issues need to follow ethical principles strictly. The use of informed consent forms with verbal consent which do not use participants' signatures is preferred by research participants in the sensitive research;

- A mixed methods approach is better for understanding the complex situations in sensitive research. Similarly, non-probability sampling method is preferred over probability sampling method due to the special nature of sensitive participants;

- The researchers need to ensure participant anonymity and confidentiality in order to involve them and readily get the responses in sensitive research;

- Support from hospital staff and person in charges or volunteers of NGOs is necessary to encourage participants in sensitive research like HIV/AIDS;

- It is important to build a good rapport with the participants and explain possible benefits of the sensitive research in order to motivate them;

- It is important not to provide monetary incentives to the participants so as to avoid biased responses and ethical conflicts; and

- All types of respondents who refused to take part in one research site should be noted and similar categories of respondents should be encouraged to take part in another research site if possible to minimize the respondents' bias.

\section{Limitations}

There are some limitations of the paper although it highlighted important issues and problems. These limitations include- concentration of the observation in limited number HIV/AIDS treatment centres and NGO shelters. Moreover, the paper is based on only observations, experience and literature reviews which hinder its generality.

\section{Competing interests}

The authors declare that they have no competing interests.

\section{Acknowledgements}

We would like to acknowledge all the participants taking part in this study. We are thankful to in-charges of all the treatment centres, research assistants and volunteers. We would like to thank president of Youth Vision (Kathmandu), Nawa Kiran Plus (Kathmandu), Chitwan Sakriya Samuha (Chitwan), Chhahari Mahila Samuha (Chitwan), and Kirat Yakthung Chumlung (KYC) (Dharan) for the support they provided during the pilot and main studies.

\section{Author E-mails}

1a.n.poudel@ljmu.ac.uk, ${ }^{2}$ dnewlands@qmu.ac.uk,

${ }^{3}$ p.p.simkhada@ljmu.ac.uk

\section{References}

1. Lee RM. Doing Research on Sensitive Topics, Sage Publication Ltd, British Library Cataloguing-in-Publication Data, Biddles Ltd, Surrey, UK, 1993, pp.-248. 
2. Sieber JE, Stanley B. 'Ethical and Professional Dimensions of Socially Sensitive Research', American Psychologist, 1988; 43(1): 49-55.

3. Brewer JD. 'Sensitivity as a Problem in Field Research: A Study of Routine Policing in Northern Ireland' AmericanBehavioural Scientist, 1990; 33:578-593. 4. Lee RM, Claire MR. 'The Problems of Researching Sensitive Topics: An Overview and introduction', American Behavioural Scientist, 1990; 33:510-528.

5. NCASC. Trainer's Guide on HIV/AIDS Orientation (For the Orientation of Medium Level Health Workers), Ministry of Health, National Centre for AIDS and STD Control, Nepal, 2000.

6. Plummer K. Documents of Life: An Introduction to the Problems and Literature of a Humanistic Method, Allen and Unwin, London, 1983.

7. Creswell JW, Klassen AC, Clark VLP, Smith KC. Best Practices for Mixed Methods Research in the Health Sciences, Office of Behavioural and Social Sciences Research (OBSSR), USA, 2011

8. Creswell JW, Clark VLP, Gutmann ML, Hanson WE. 'Advanced Mixed Methods Research Designs', In: Research Design (Ed.: DD Vaus). Sage Publication, British Library Cataloguing-in-Publication Data. The Bardwell Press, UK, 2006, pp. 249.

9. Banteyerga H, Kidanu A, Nyblade L, MacQuarrie, Pande R. Exploring HIV and AIDS Stigma and Related Discrimination in Ethiopia: Causes, Manifestations, Consequences and Coping Mechanisms,Miz-Hasab Research Center, International Center for Research on Women, Ethiopia, 2004.

10. Hires KA. Predictors of HIV/AIDS Related Stigma and Discrimination among Anglophone Caribbean Women, Open Access Dissertations, Paper 912, 2012.

11. Lambda Legal. HIV Stigma and Discrimination in the U.S.: An EvidenceBased Report, Lambda Legal: making the case for equality, USA, 2010.

12. Valerio MA, Mainieri T. 'EthicalPrinciples'. In: Encyclopaedia of Survey Research Methods (PJ Lavrakas, Ed.), Thousand Oaks, Sage Publications, Canada, 2008.

13. Erlen JA, Sauder RJ, Mellors MP. 'Incentives in Research: Ethical Issues', Orthopaedic Nursing, 1999; 18 (2): 84-87.

14. Macklin R. 'On Paying Money to Research Subjects: 'Due' and 'Undue' Inducements', Institutional Review Board, 1981; 3: 1-6.

15. Gomm R. Social Research Methodology: A Critical Introduction, Palgrave Macmillan, Library of Congress Cataloguing-in-Publication Data, New York, 2004, pp. 350

16. Baker TL. Doing Social Research, McGraw-Hill Inc., Library of Congress Cataloguing-in-Publication Data, Mexico, 1988, pp-483.

17. Courser M. 'Consent Form', In: Encyclopaedia of Survey Research Methods [Ed.: PJ Lavrakas], Sage Publication, Library of Congress Cataloguing-in-Publication Data, London, 2008, pp. 132-133.

18. Herek GM, Capitanio JP. 'AIDS Stigma and Sexual Prejudice', American Behaviour Science, 1999; 42:1126-1143.

19. Manijsin T, Sangyos D, Pirom P. Families and Communities Living with AIDS, Ban Head District, KhonKaen Province, Thailand, 2003.

20. Sweat MD, Levin M. 'HIV/AIDS Knowledge among the U.S Population', AIDS Education and Prevention, 1995; 7: 355-372.

21. Stutterhein SE, Pryor JB, Bos AER, Hoogendijk R, Muris P, Schaelma HP. 'HIV-related Stigma and Psychological Distress: the Harmful Effects of Specific Stigma Manifestations in Various Social Settings', AIDS, 2009; 23:2353-2357. 22. Bos AE, Schaalma HP, Pryor JB. 'Reducing AIDS-related Stigma in Developing Countries: the Importance of theory and Evidence-based Interventions', Psychology, Health \& Medicine, 2008; 13:450-460.

23. Mahajan AP, Sayles JN, Patel VA, Remien RH, Wawires SR, Ortiz DJ et al. 'Stigma in the HIV/AIDS Epidemic: A Review of the Literature and Recommendations from the Way forward' AIDS, 2008; 22 (S2): S67-S79.

24. Parker R, Aggleton P. 'HIV and AIDS-Related Stigma and Discrimination: A Conceptual Framework and Implications for Action', Social Science \& Medicines, 2003; 57:13-24.

25. Wasti SP, Simkhada P, Randall J, Teijlingen E. 'Issues and Challenges of HIV/ AIDS Prevention and Treatment Programme in Nepal', Global Journal of Health Science, 2009;1(2): 62-72.

26. Wilkinson M, Moore A. 'Inducement in Research', Bioethics, 1997; 11:373389.

27. Grady C. 'Money for Research Participation: Does it Jeopardize Informed Consent?' American Journal of Bioethics, 2001; 1:40-44.

28. Bentley JP, Thacker PG. 'The Influence of Risk and Monetary Payment on the Research Participation Decision Making Process', Journal of Medical Ethics, 2004; 30:293-298.

29. Gates S, Williams MA, Withers E, Mt-Isa S, Lamb SE. 'Does a Monetary Incentive Improve the Response to a Postal Questionnaire in a Randomised Controlled Trial? The MINT Incentive Study', Biomedical Journal, 2009; Accessed from: http://www.trialsjournal.com/content/10/1/44 [Accessed on 15 Feb 2013].

30. Russell ML, Moralejo DG, Burgess D. 'Paying Research Subjects: 'Partici- pants Perspectives', Journal of Medical Ethics, 2000; 26:126-130.

31. Massey DS, Tourangeau R, Singer E, Ye C. 'The Use and Effects of Incentive in Surveys, The ANNALS of the American Academy of Political and Social Science, 2013; 645 (1):112-141.

32. Palmer WE. 'Monetary Inducement to Research Participation', Pharos Alpha Omega Alpha Honor, Medical Society, 1985; 48: 26-30.

33. Vaus DA de. Surveys in Social Research, Allen and Unwin, British Library Cataloguing-in-Publication Data, Athenaeum Press, Newcastle, 1993.

34. Kennedy J. 'Confidentiality', In: Encyclopaedia of Survey Research Methods [Ed.: P.J. Lavrakas]. Sage Publication, Library of Congress Cataloguing-in-Publication Data, London, 2008a, pp 131-132.

35. Knight PT. Small-Scale Research, Sage Publications Ltd, British Library Cataloguing in Publication Data, London, 2002, pp.-223.

36. Kennedy J. 'Anonymity', In: Encyclopaedia of Survey Research Methods [Ed.: PJ Lavrakas]. Sage Publication, Library of Congress Cataloguing-in-Publication Data, London, 2008b, pp. 27-28.

37. The Kathmandu Post. AIDS Fund Only via Govt Now [Online] [24 January 2011], From: From: http://www.ekantipur.com/the-kathmandupost/2011/01/24/nation/aids-funds-only-via-govt-now/217615.html [Accessed on 25 July 2012]. 